\title{
El tratamiento antihipertensivo basado en monitoreo ambulatorio de la presión arterial disminuye el uso de medicación pero no ahorra costos.
}

Antihypertensive treatment based on conventional or ambulatory blood pressure measurement. A randomized controlled trial. Staessen JA, Byttebier G, Buntinx F, Celis H, O’Brien ET, Fagard R. JAMA 1997:278:1065-1672.

\section{Objetivo}

Comparar la eficacia de la medición de la presión arterial (TA) en el consultorio con el monitoreo ambulatorio en el manejo de los pacientes hipertensos.

Diseño

Estudio multicéntrico, randomizado y de grupos paralelos.

Lugar

Cincuenta y seis centros de hospitales universitarios y regionales en Bélgica, Irlanda, Holanda y Luxemburgo. Médicos de familia y médicos clínicos de hospitales universitarios y regionales.

\section{Pacientes}

Participaron 419 pacientes mayores de 18 años con TA diastólica promedio $>95 \mathrm{mmHg}$ medida en consultorio (MC). Se los randomizó en dos grupos de acuerdo a la estrategia de manejo: 1 . Basado en MC; 2. Basado en monitoreo ambulatorio de presión arterial (MAPA).

\section{Intervención}

El tratamiento con drogas antihipertensivas fue ajustado en forma escalonada guiado por el promedio de la TA medida con MAPA $(n=213)$ o en el promedio de 3 registros de TA diastólica en posición sentado $(n=206)$ medida en consultorio. Si la TA diastólica estaba por encima (>89 mm Hg), dentro ( $80-89 \mathrm{mmHg}$ ) o por debajo (<80 mm Hg) de los objetivos, un médico ciego a la randomización de los pacientes, intensificaba el tratamiento, lo mantenía igual o lo reducía.

\section{Medición de resultados principales}

Se midieron los niveles de la TA logrados por cada uno de los grupos. Se registró la intensidad del tratamiento farmacológico, los cambios electrocardiográficos y la masa ventricular izquierda medida por ecocardiograma, así como los síntomas reportados a través de un cuestionario y los costos.

\section{Resultados}

El seguimiento medio fue de 182 días (rango 5-95, 85-258). Se pudo suspender el tratamiento en más pacientes del grupo MAPA que en el grupo MC $(26,3 \%$ vs $7,3 \% ; P<0,001)$. En el grupo MAPA hubo menor necesidad de tratamiento con múltiples drogas que en el MC $(27,2 \%$ vs $42,7 \% ; P<0,001)$. El grupo MC alcanzó una TA marginalmente menor que el MAPA al final del estudio sólo si se la medía por MAPA $(p<0.05)$. La masa ventricular izquierda y los síntomas referidos fueron similares en los 2 grupos. Los potenciales ahorros en el grupo con medición de la TA en términos de un tratamiento farmacológico menos intensivo y menos visitas al médico fueron compensados por los costos del MAPA.

\section{Conclusiones}

El ajuste del tratamiento antihipertensivo basado en el MAPA en lugar de las mediciones de consultorio permiten un tratamiento farmacológico menos intensivo. No hay diferencias relevantes en cuanto al control de la TA, la sensación de bienestar general, la hipertrofia ventricular izquierda, y a los costos totales del tratamiento antihipertensivo.

\section{COMENTARIO}

Los estudios epidemiológicos muestran que los pacientes con hipertensión arterial (HTA) bien controlada representan una pequeña proporción de la población de hipertensos (1-4). Esto puede ser real, debido a una mala adherencia al tratamiento antihipertensivo; o ficticio, ya que los pacientes con HTA controlada pueden tener registros elevados en el consultorio. Esto último se conoce como efecto de guardapolvo blanco (5-6); se observa más en ancianos y no tiene relación con la severidad de la HTA. Los hipertensos que presentan este efecto no tienen peor pronóstico que los que no lo presentan, como lo demostró el estudio PIUMA (7), por lo que se lo considera benigno. Una entidad diferente es la Hipertensión de guardapolvo blanco, también llamada HTA aislada de consultorio. Esta se diagnostica en pacientes que tienen la TA elevada exclusivamente en el consultorio. La prevalencia es entre el 10 y $20 \%$ de todos los hipertensos y disminuye con la gravedad de la HTA (8). Como los estudios que evaluaron la efectividad a largo plazo del tratamiento antihipertensivo se guiaron por las mediciones en el consultorio, las normas de manejo de la HTA de guardapolvo blanco son las mismas que para el resto de los hipertensos (1-2).

EL MAPA es útil en pacientes con sospecha de HTA de guardapolvo blanco, en pacientes con HTA resistente, en los que presentan síntomas de hipotensión arterial con medicación antihipertensiva, HTA episódica y en sujetos con disfunción autonómica (1-2).

A pesar del incremento en la utilización del MAPA, aún no se ha determinado como afecta al tratamiento de la HTA. En este estudio, con el uso del MAPA, el tratamiento antihipertensivo pudo ser evitado en el $25 \%$ de los pacientes (con efecto de guardapolvo blanco) y se evitó el tratamiento con múltiples drogas en el 15\%. En conclusión, este estudio muestra que manejar la HTA basándose en el MAPA utiliza menos medicación a costos similares. Queda por definir la evaluación comparativa de estas estrategias a largo plazo.

Dr. Gabriel Waisman Unidad de Fisiología Clínica e Hipertensión Arterial. Servicio de Clínica Médica. Hospital Italiano de Buenos Aires

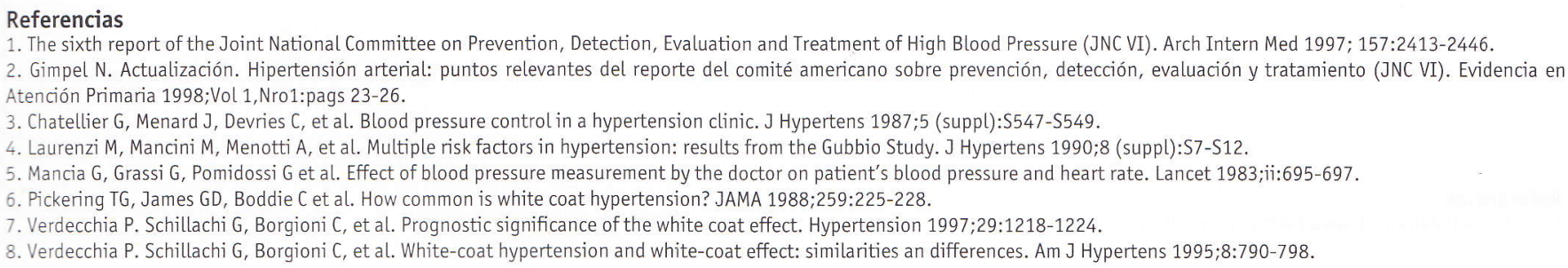

